\title{
Design of a phase shifting interferometer in the EUV for high precision metrology.
}

\author{
María Gabriela Capeluto, ${ }^{1,2, *}$ Mario Carlos Marconi, ${ }^{3}$ and Claudio Cesar lemmi ${ }^{1}$ \\ ${ }^{1}$ Departamento de Física, Facultad de Ciencias Exactas y Naturales. Universidad de Buenos Aires. Pabellón 1, \\ Ciudad Universitaria, Buenos Aires, Argentina. \\ ${ }^{2}$ Instituto de Física de Buenos Aires (IFIBA) - CONICET - Universidad de Buenos Aires, Argentina. \\ ${ }^{3}$ Department of Electrical and Computer Engineering, \\ and NSF ERC for Extreme Ultraviolet Science 83 Technology, Colorado State University, USA.
}

compiled: October 9, 2013

\begin{abstract}
The design of a phase shift interferometer in the extreme ultraviolet (EUV) is described. The interferometer is expected to achieve a significant higher precision as compared with similar instruments that utilize lasers in the visible range. The interferometer's design is specifically adapted for its utilization with a table top pulsed capillary discharge extreme ultraviolet laser. The numerical model evaluates the errors in the interferograms and in the retrieved wavefront induced by the shot-to-shot fluctuations and pointing instabilities of the laser.
\end{abstract}

OCIS codes: (050.5080) Phase shift; (140.7240) UV, EUV, and X-ray lasers; (120.3180y) Interferometry

\section{Introduction.}

The significant progress in the development of compact light sources with wavelengths in the extreme ultraviolet (EUV), covering the range of 10 to $50 \mathrm{~nm}$ $[1,2]$, has fostered research in several fields that use short wavelengths. Some examples are the characterization and processing of materials [3-6], techniques for high resolution metrology [7-11], studies in atomic physics, photochemistry and photophysics [12, 13], biological imaging [14-17], the diagnosis of very high-density plasmas [18-21], the study of nonlinear phenomena [22, 23], and even integrated circuit lithography [24].

The development of coherent sources in the EUV has allowed the implementation of interferometers with improved resolution as compared with those in the visible range. However, at shorter wavelengths the implementation is more complicated due to the lack of high efficiency optics. Common path interferometers, such as the shear interferometer [25] and the point-diffraction interferometer (PDI) [7], were demonstrated at EUV wavelengths

\footnotetext{
* Corresponding author: maga@df.uba.ar
}

for high resolution metrology. Although the standard metrology schemes that use a single interferogram are very effective, these methods have several limitations. Since they are based on localization of the centres of the fringes, there is a compromise between the accuracy and the sampling density in the image. Moreover, most of them operate in conditions of great tilt between the interfering beams, so it is necessary the addition of a spatial carrier frequency to remove ambiguities in the retrieved phase and determine the overall sign or polarity of the wavefront (i.e. whether it is concave or convex).

The phase shifting interferometry (PSI) overcomes several of the problems present in single interferogram methods. Using multiple interferograms the phase of the wavefront can be retrieved at each point in the space domain regardless of the neighbouring points by a simple calculation. In this case it is not necessary to add a spatial carrier frequency or locate the centre of the fringes, and also the polarity of the wavefront is unambiguously determined. Moreover, the possibility of evaluating the phase at every point increases the interferogram spatial resolution.

Medecki et al developed a PSI compatible with EUV light illumination $[9,11]$. This interferometer 
is based on the PDI, with the additional ability to implement the phase shifting algorithm, and consequently improving significantly its performance. In this EUV interferometer, a reference spherical wavefront beam is generated by diffraction in a small pin-hole, therefore no reference surface is needed reducing significantly the sources of error, which allows obtaining an accuracy of the spherical reference beam better of a fraction of the wavelength $\left(0.038 \mathrm{~nm}\right.$ for $\left.\lambda_{E U V}=13.5 \mathrm{~nm}\right)$ [11]. Despite this, the layout of this device imposes a large separation of the two beams that interfere. As a result, the interference pattern contains information not only from the object to be measured, but also a large amount of tilt and coma in the direction of the separation of the beams, which appears as a systematic error in the measurements.

In this article an alternative design of a EUV interferometer is presented. The feasibility of using this interferometer to implement a phase shift algorithm with a pulsed compact EUV laser as illumination is analyzed. This study is based on a beam propagation code that takes into account the main characteristics of the optical system and the EUV laser source.

We present a complete analysis of the expected performance of the instrument, with emphasis on the utilization of a EUV capillary discharge laser. This laser emits at a wavelength of $46.9 \mathrm{~nm}$, and is well adapted to inspect substrates or multilayered optics centered at this wavelength. Also, thin samples with reasonably low absorption are suitable specimens that can be inspected with this instrument at this particular wavelength. However, the calculation presented here are more general and can be applied to other sources, like shorter wavelength lasers and HHG sources, that are now capable to emit below $10 \mathrm{~nm}$, where the absorbance of most materials is drastically reduced. For these alternative sources the spectrum of applications broadens. Samples can be inspected when placed in one of the branches of the interferometer. The interferometer is a Mach-Zehnder configuration that allows a large working space and in principle will be well adapted to analyze for example biological samples.

The light source considered in this design is a table top capillary discharge laser. It produces an intense beam at $\lambda=46.9 \mathrm{~nm}$ with pulses of approximately $400 \mu \mathrm{J}$ at repetition rates up to $4 \mathrm{~Hz}$ $[26,27]$. The beam has a high degree of spatial coherence that varies with the length of the gain medium [28]. The laser has also a narrow spectral bandwidth, $\Delta \lambda / \lambda \approx 3-4 \times 10^{-5}[29]$, corresponding to a temporal coherence length of $700 \mu \mathrm{m}[28,30]$. The high degree of spatial coherence of the capillary discharge laser allows for a fully coherent illumination of the instrument. If other EUV sources are used, it should be required a coherence area at the input of the interferometer larger than the entrance pupil of the instrument.

The implementation of an interferometer with $46.9 \mathrm{~nm}$ illumination utilizing this highly coherent laser source would provide a high precision metrology tool. However, since this laser medium is a hot plasma produced by a fast pulsed discharge, it has inherently variations in the position of the column that may generate a drift of the laser beam from shot to shot. This in turn may produce a pointing instability in a PSI setup that influences the phase retrieving. In this paper the influence of this effect in the performance of the instrument is analyzed and the limitations of the system are determined.

The paper is organized as follows: The design of the interferometer is described in section 2 . The beam propagation through the interferometer is studied in section 3 . In section 4 , the performance of the interferometer, as a function of the fluctuations in the laser illumination, is analyzed by numerical simulations. Finally, the conclusions are presented in section 5 .

\section{Design of the interferometer.}

One limitation for an instrument designed to operate at $\lambda=46.9 \mathrm{~nm}$ is the lack of refractive optics. Thus the design is restricted to consider only diffractive (Fresnel zone-plates and diffraction gratings) and reflective optics (multilayer or grazing incidence mirrors).

When implementing the PSI technique with accuracy of the order of a fraction of the wavelength, it is convenient that the wavefronts of the interfering beams are to be as similar as possible. Since interferometry is a comparative subtractive technique, it is desirable that the obtained phase difference corresponds only to the phase introduced by the tested object, and does not include any spurious phase due to the geometry of the interferometer. From this point of view, although the point diffraction interferometer developed by Medeki et al [9] is suitable for using EUV illumination, it introduces errors due to its layout (for example, tilt and coma), which should be compensated in order to increase the accuracy of the measurement [8]. Another alternative design was proposed by Chilla et al [31], which was subsequently used for the study of temporal evolution of the electron density in laser-generated plasma $[18,32]$. The configuration of the interfer- 
ometer described by Chilla introduces an angular dispersion in one of the beams that produces high astigmatism, making the interfering beams very different.

In order to partially overcome these problems, the interferometer configuration shown in figure 1 was choosen. It is similar to the one described by Chilla [31], but it uses transmission gratings instead of reflective gratings. This scheme makes the optical path of the two beams completely symmetric thus eliminating the astigmatism. The output beams are collinear so the tilt and coma are also minimized. The incident beam impinges normally on the first transmission diffraction grating $G_{1}$ that splits the incident wavefront in the multiple diffraction orders. The first positive and negative diffraction orders (1 and -1) define the two arms of the interferometer. The zero diffraction order is blocked. The two beams are reflected in two mirrors that redirect them towards a second diffraction grating $G_{2}$ identical to the first one. This symmetric layout makes the incident angles on $G_{2}$ equal to the diffraction angles on $G_{1}$ recombining the beams in a collinear path. The grating $G_{1}$ is situated at a distance $d_{o}$ from the laser output. The distance between $G_{1}$ and $G_{2}$ is $\mathrm{G}$, and the two mirrors are located at half way between the two gratings. After the second grating, the detector is situated at a distance $z_{d_{o}}$. Considering normal incidence on the grating $G_{1}$, the angles at which the order 1 and -1 are diffracted, are defined by the grating equation

$$
\sin \left(\theta_{ \pm}^{o}\right)= \pm \frac{\lambda}{p}
$$

where $\lambda$ is the wavelength and $p$ is the period of the diffraction grating.

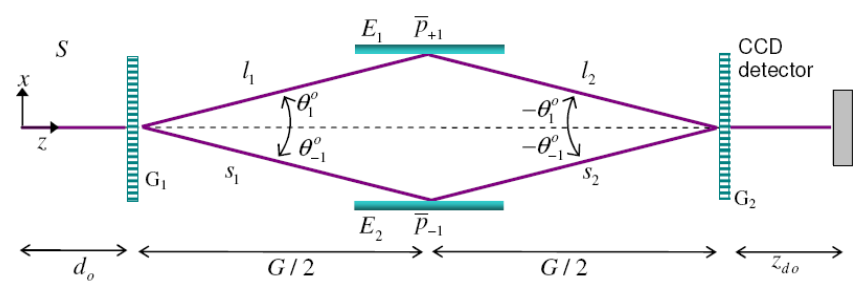

Fig. 1. Schematic of the interferometer. The gratings G1 and G2 are used as beam splitters. The mirrors (E1 and E2) direct the beams to impinge at the same angle on G2. The parameters are described in the text.

If the grating is displaced transversally to the grooves to introduce the phase changes needed to implement the PSI technique, the distances be- tween the components of the interferometer remain constant.

A singular aspect of the design described in this paper is that it contemplates the utilization of a pulsed plasma EUV laser source. Thus, the PSI technique must be implemented with interferograms acquired with different pulses. Due to the particular dynamics of the generation of the laser, there is a possibility that the direction of the beam slightly changes from shot to shot due to natural fluctuations in the location of the plasma column. Although this is not important for several of the applications demonstrated so far with this laser $[19,20,33-38]$, this is an aspect that must be taken into account when designing a high resolution instrument like a PSI interferometer. This small variation in the pointing direction of the laser beam imposes a source of error for this particular application that has to be evaluated. It will be especially considered in the model that follows.

The most appropriate kind of phase retrieving algorithms for our source is that used by the phase stepping technique which introduces discrete phase shifts in the reference arm (one step for each pulse). The recovery of the phase requires at least three interferograms. Usually, by increasing the number of interferograms it is possible to reduce the error in the evaluation of the wavefront. However, for the specific design of this instrument, assuming that the illumination can have a random small fluctuation in the incidence angle, increasing the number of interferograms adds a random noise that limits the accuracy in the phase retrieval process. To establish a trade off between the number of interferograms and the noise the four bucket algorithm [39] was used, which is a significant improvement over the three step algorithm [40, 41]

The intensity distribution for two beam interference considering a bucket algorithm is given by

$$
I_{n}(\bar{x})=I_{o}(\bar{x})\left\{1+v(\bar{x}) \cos \left(\Omega(\bar{x})+\delta_{n}\right)\right\}
$$

where $\delta_{n}$ are the discrete shifts introduced in $n$ steps, $I_{o}(\bar{x})$ is the incoherent sum of the intensities of the two interfering beams, $v(\bar{x})$ is the contrast and $\Omega(\bar{x})$ is the unknown phase difference. In the four bucket technique $[40,42]$, the phase shifts $\delta_{n}$ are $0, \pi / 2, \pi$ and $3 \pi / 2$, so the four corresponding intensities are obtained by the substitution of this values in equation 2. Operating with the four intensities, it is possible to get the spatial phase distribution in modulus $2 \pi$ 


$$
\Omega(\bar{x})=\tan ^{-1}\left[\frac{\left.I_{4}(\bar{x})-I_{2}(\bar{x})\right)}{I_{1}(\bar{x})-I_{3}(\bar{x})}\right] .
$$

The most common errors such as detector non linearities, or the introduction of spurious phase shifts due to the inaccuracy of the mechanical components (translation units), or non linearities of the piezoelectric actuator which is normally used to move the mechanical components, are largely analyzed in the literature [43]. Most of the algorithms are designed to compensate and reduce the susceptibility to these errors. In the case analyzed here, the largest source of error is given by the fact that successive interferograms are recorded from different laser pulses. An error analysis due to pointing instabilities in the incident beam has not been yet done, since in general continuous wave stabilized lasers with high pointing stability are used for this type of interferometer. Since those are not the characteristics that EUV laser, it is interesting to analyze what are the tolerances on these parameters that allow an accurate phase retrieval.

\section{Beam propagation through the interferome- ter.}

In this section the propagation of laser beams through the interferometer is modelled, in the general case where the incidence angle on the first grating $(\gamma)$ and the position of the incidence axis $\left(x_{o}, y_{o}\right)$ vary relative to the instrument axis due to the pointing fluctuations of the illumination beam. Figure 2 exemplifies the ray tracing through the interferometer for two cases where there are fluctuations in the incidence angle and in the incidence axis and compares them with the case where there is full pointing stability, and so $\gamma=0$ and $\left(x_{o}, y_{o}\right)=(0,0)$. It is evident that it is necessary to evaluate the influence of the fluctuation of the parameters $\{\gamma, x o, y o\}$ on the retrieved phases since each interferogram has an additional random phase that is originated by these fluctuations. The displacements of the incident beam in the direction $\hat{y}$ (parallel to the grating lines) have no significant effect on the phase. Thus all the deviations caused by the fluctuations should be considered in the plane $x z$ of the laboratory system.

The transversal intensity distribution of the laser has an annular shape, and its overall behaviour (collimation, divergence, and transverse size) can be adequately described by the lower order Gaussian beam mode $\left(T E M_{o o}\right)[44,45]$. This is a good approximation to calculate the propagation of the

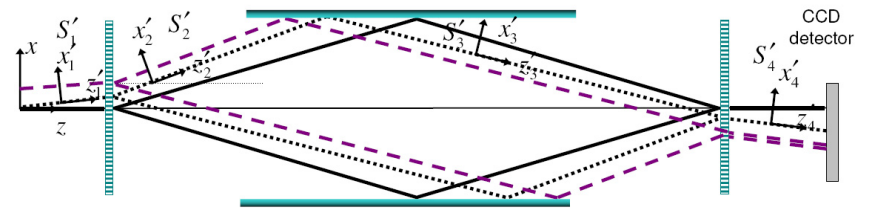

Fig. 2. Ray tracing indicating the posibles pointing stability problems. Full pointing stability (solid line), error in the incidence angle (dotted line), error in the incidence angle and displacement of the emission axis (dashed line)

beam in the interferometer and to obtain analytical expressions for the interference patterns under more realistic conditions than considering the propagation of plane waves.

The electric field at the input of the interferometer is considered to be a spatial Gaussian function with a narrow spectral distribution, with an incidence angle $\gamma$ and a beam waist $\sigma$ located on $\left(x_{o}, y_{o}, 0\right)$. The propagation of the Gaussian beam is performed in the natural coordinate system $S^{\prime}$ in which the direction of propagation is parallel to the axis $z^{\prime}$, or the deviations are small enough to satisfy the paraxial approximation. In this way in the region of $\mathrm{z}$ varying form 0 to $d_{o}$, where the first grating is placed, the beam propagates parallel to the axis $z_{1}^{\prime}$ in the coordinate system $S_{1}^{\prime}$, which is the natural Gaussian system. The coordinate $\hat{z}_{1}^{\prime}$, is oriented at an angle $\gamma$ respect to the coordinate $\hat{z}$ of the laboratory system $S$. Assuming that the origin of $S$ and $S_{1}^{\prime}$ coincides with the output of the laser, a beam that exits the laser at a distance $x_{o}$ and an angle $\gamma$ from the $z$ axis, will propagate in the direction $z_{1}^{\prime}$ of the system $S_{1}^{\prime}$, with its intensity maxima in the position given by $x_{o}^{\prime}=x_{o} \cos (\gamma)$, $z_{o}^{\prime}=x_{o} \sin (\gamma)$ and $y_{o}^{\prime}=y_{o}$.

The Kirchhoff integral, in the Fresnel approximation, was used to describe the free propagation of the beams. In figure 2 the beam paths were traced for values of $\left\{\gamma, x_{o}, y_{o}\right\}$ much larger than expected for clarity. The distances that the beams propagate are calculated using geometric analysis of their trajectories and the effect of the gratings and mirrors are expressed in the Gaussian beam formalism, according to the model developed by Martinez [46]. This model allows for the inclusion of the effects of the angular dispersion $\alpha$ in the grating and the chromatic dispersion $\beta$ due to the finite bandwidth of the beam.

After the diffraction at the gratings and reflections on the mirrors, each arm of the interferometer is expressed in its own reference system 
that changes orientation as the beam propagates through the interferometer. A different arabic numeral is used to indicate the natural system of the Gaussian beam in each region of the interferometer as shown in figure 2 . So $S_{1}^{\prime}$ is the natural system of the Gaussian beam between the beam waist and the first grating, $S_{2}^{\prime}$ is the natural system between the first grating and the mirror, $S_{3}^{\prime}$ corresponds to the section between the mirror and the second grating and finally $S_{4}^{\prime}$ to the section between second grating and the detector. The Kirchhoff-Fresnel integrals are evaluated in the corresponding natural coordinate system $S_{i}^{\prime}$ for each segment of the propagation in the interferometer. The two beams are finally written in the laboratory system to calculate the interferogram. This is done by an appropriate transformation of coordinates, composed by a translation and a rotation.

As it is shown in figure $1, l_{1}$ and $l_{2}$ are the path lengths in the systems $S_{2}^{\prime}$ and $S_{3}^{\prime}$ of the arm 1 respectively. Analogously, $s_{1}$ and $s_{2}$ are the path lengths in the systems $S_{2}^{\prime}$ and $S_{3}^{\prime}$ of the arm -1 respectively. These magnitudes and the angles at which the beams emerges from the interferometer $\left(\theta_{ \pm 1}^{G_{2}}\right)$ are calculated as a function of the incidence angle and the impinging point on the first grating of the interferometer $\left\{\gamma, x_{o}, y_{o}\right\}$, the construction parameters $\left(d_{o}, G, z_{D}, p\right)$ and the tilt $\varphi$ of the mirror in the test arm.

The phase shifts needed to implement the algorithm are introduced through the translation of one of the gratings of the interferometer. A translation of a diffraction grating by a distance $\delta$ with respect to the $z$ axis of the coordinate system, introduce a phase $\phi_{n}^{S}=2 \pi \delta n / p$ in the diffraction order $n$, where $p$ is the grating period. Similarly, if the alignment of the incident beam produces a beam that impinges at a distance $x^{G_{1}}$ from the interferometer axis on $G_{1}$ and, after the propagation, impinges at a distance $x^{G_{2}}$ from the interferometer axis on $G_{2}$, a phase shift $\varphi_{n}=-2 \pi\left(x^{G_{1}}+x^{G_{2}}\right) n / p$ is introduced in the order $n$. The quantities $x^{G_{1}}$ and $x^{G_{2}}$ are calculated by geometrical optics considerations for each arm of the interferometer. Then, the total phase that is added due to the gratings positioning relative to the beam is $\phi_{n}=\left(\delta-x^{G_{1}}-x^{G_{2}}\right) 2 \pi n / p$ with $n= \pm 1$ for each arm of the interferometer.

The expression for the electric field at the output of the interferometer is then:

$$
A(\bar{x},\{\bar{p}\}, \omega)=A_{1}\left(\bar{x}, l_{2}, l_{1}, d, \omega\right)+A_{2}\left(\bar{x}, s_{2}, s_{1}, d, \omega\right) .
$$

sions for the electric field of the arm 1 and -1 respectively and they depend on the coordinates of the system $S$ at the measurement plane $\bar{x}=(x, y, z)$, the optical paths through the arms, the propagation distance $d$ in the system $S_{1}^{\prime}$, and the frequency $\omega$ of the electric field (see appendix for complete expressions). All the distances are dependant on the parameters $\{\bar{p}\}=\left\{\gamma, x_{o}, y_{o}, \varphi, d_{o}, G, z_{d o}, p\right\}$ - The intensity at the detection plane is $I(\bar{x}) \propto$ $\frac{c \varepsilon_{o}}{2} \int_{-\infty}^{\infty}|A(\bar{x},\{\bar{p}\}, \omega)|^{2} d \omega$.

The last elements of the design are a telescope to collimate the incident beam and an imaging system in the output to rely the plane where is placed the object under study on the detector's plane.

The telescope is situated at the entrance of the interferometer and can be implemented with two curve mirrors or two zone plates. For the calculation purposes it will be considered that the telescope is composed of these elements with focal distances $f_{1}$ and $f_{2}$, and a distance between them equal to the sum of the focal distances.

Let us suppose that the Gaussian incident beam on the telescope is characterized by a complex radius of curvature $q(z)$ having a waist $\sigma$ and the divergence $\theta$. The distance between the beam waist and the grating $G_{1}$ is fixed at $d_{o}$ so $d_{o}=$ $a+f_{1}+f_{2}+c$. Assuming that the telescope is composed of mirrors and using ABCD matrix formalism $[44,45]$, the complex radius of curvature $q^{\prime}(z)$ of the beam at the exit of the optical system is given by $q^{\prime}(z)=a\left(f_{2} / f_{1}\right)^{2}-\left(f_{2}+f_{1}\right)\left(f_{2} / f_{1}\right)+c+i z_{r}\left(f_{2} / f_{1}\right)^{2}$. This represents a Gaussian beam that propagates a distance $d_{o}^{\prime}=a\left(f_{2} / f_{1}\right)^{2}-\left(f_{2}+f_{1}\right)\left(f_{2} / f_{1}\right)+c$ to the grating $G_{1}$ and with a confocal parameter given by $z_{r}^{\prime}=z_{r}\left(f_{2} / f_{1}\right)^{2}$. Now the new beam waist $\sigma^{\prime}$ is calculated considering the confocal parameter

$$
z_{r}^{\prime}=\frac{\pi \sigma^{\prime 2}}{\lambda} \Rightarrow \sigma^{\prime}=\sigma\left(f_{2} / f_{1}\right),
$$

with a divergence given by

$$
\varsigma^{\prime}=\frac{2 \lambda}{\pi \sigma^{\prime}} \Rightarrow \varsigma^{\prime}=\varsigma\left(f_{1} / f_{2}\right)
$$

Thus, to consider the effect of the telescope it is equivalent to considered that the input beam in the interferometer is a Gaussian beam with a waist $\sigma^{\prime}$, given by equation 5 , and a divergence $\varsigma^{\prime}$ given by equation 6 , and that propagates a distance $d_{o}^{\prime}$ from the position of the waist.

The optical surface under inspection is placed on the mirror $E_{1}$ of the test arm. Thus, to evaluate 
the wavefront distortion at that position it is necessary to use an optical system that images the object plane onto the detection plane. Adding the optical system will introduce a phase term common to the object and reference beams. The objects inspected by interferometric techniques are expected to introduce a small dephasing in the object beam, and not produce a significantly large misalignment of the instrument. Thus an alignment of the instrument close to the zero fringe will remove the linear phase and the global phase (piston term) components of the Zernike expansion. This can also be done afterwards with an adequate processing of the interferogram. To avoid including extra calculations and keeping in mind that the objective of this analysis is to evaluate the requirements on the laser pointing stability to implement the phase shift technique, a simplified approach is followed. The field distribution just after the second grating given by its expression in the system $S$ evaluated in $z=G+d_{o}$, was back propagated a distance corresponding to the position in which the object is located.

Finally, it is useful to estimate the throughput of the interferometer and the minimum requirements in energy in the input of the instrument. If we assume that 1000 counts per pixel in the CCD are required to overcome the thermal noise, it is possible to find the minimum energy requirements for the source. Taking into account the efficiency of the different components (diffraction efficiency in the first order $\sim 0.1$ for a grating, reflectivity of the grazing incidence mirrors $\sim 0.96$, efficiency in the first order for a zoneplate $\sim 5 \%$ ) the total throughput of the interferometer is estimated to be of the order of $2 \times 2.4 \times 10^{-7}$, where the number 2 accounts for the two beams that are interfering. Since each photon would produce 26 electrons in the CCD for the $46.9 \mathrm{~nm}$ laser, then the total energy at the input of the interferometer should be $70 \mu \mathrm{J}$, which is still several times smaller than the energy available with the $46 \mathrm{~nm}$ laser.

In the next section, the numerical simulations that allowed for the evaluation of the tolerance of the phase retrieval algorithm to the fluctuation of the pointing of the illumination laser are described.

\section{Numerical simulations and analysis of the phase measurement.}

In this section numerical simulations of the phase retrieval calculation are presented. For this simulation we introduced a test object consisting of a wedge with a reflecting surface that forms an angle $\varphi$ with respect to a reference plane placed in the position of one of the mirrors of the interfer- ometer. The reference plane is parallel to the surface of the mirror $E_{1}$ (figure 1). The other folding mirror of the interferometer $\left(E_{2}\right)$ acts as the reference surface. Ideally, the test object depicted in the figure 3 would introduce a linear phase in the reflected wavefront only in the region of the wedge. Thus, when the interferometer is perfectly aligned and there is no random variation in the alignment of the illumination beam, no interference fringes are produced in the region corresponding to the reference plane, while equally spaced straight fringes will be formed in the region corresponding to the wedge. Once the phase is retrieved, after measuring the four interferograms necessary to implement the phase stepping algorithm, the wavefront introduced by this object should be properly reconstructed. However if random fluctuations in the direction of the laser are taken into account, the phase reconstruction will produce random variations in the phase.
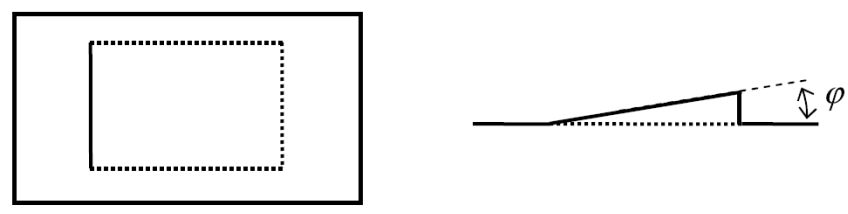

Fig. 3. Scheme of the object utilized to evaluate the stability of the phase recovery process, the object is composed of a wedge tilted in an angle $\varphi$ located on a plane surface, parallel to one of the mirrors of the interferometer

To perform the numerical simulation realistic typical values of the interferometer dimensions were assigned. The period of the gratings $p$ is assumed to be equal to $1 \mu \mathrm{m}$, the distance between the laser and the first grating and the distance between gratings were chosen as $660 \mathrm{~mm}$, and $G \sim 640 \mathrm{~mm}$ respectively, and the focal distances of the mirrors or zone plates that build up the telescope were $f_{1}=10 \mathrm{~mm}$ and $f_{2}=500 \mathrm{~mm}$. The distances between the telescope, the capillary laser and the first grating, were chosen in order to keep the distance $d_{o}$ between the beam waist (at the capillary output) and the first grating $G_{1}$, fixed at a value of $660 \mathrm{~mm}$. The tilt of the wedge was set $\varphi=5 \times 10^{-6} \mathrm{rad}$, which gives rise to three to four fringes in the interferogram.

In the four step phase shifting algorithm (equation 3), four interferograms are required which are acquired with four different laser pulses. The following analysis assesses the influence of the typical shot-to-shot variations (pointing instabilities and incidence angle) in the process of retrieving the 
phase of the object beam. To evaluate this influence, let us consider that the pulse $n$, corresponding to a phase shift $\delta_{n}$ is associated with a set of laser fluctuation parameters, indicated as $\left\{\gamma_{n}, x_{o n}, y_{o n}\right\}$ that are the incidence angle and the impinging point on the first grating of the interferometer. Each intensity distribution necessary for the implementation of the algorithm in equation 3 is defined as: $I_{n}(\bar{x})=I\left(\bar{x},\left\{\gamma_{n}, x_{o n}, y_{o n}\right\}, d_{o}, G, z, \varphi, \delta_{n}, \omega\right)$ for $n=1$ to 4 , where the dependence parameters is indicated explicitly. Thus, the retrieved phase depends on the fluctuating parameters $\left\{\gamma_{n}, x_{o n}, y_{o n}\right\}$ through the four required intensity distributions as $\Omega(\bar{x})=\Omega\left(\bar{x},\left\{\gamma_{n}, x_{o n}, y_{o n}\right\}_{n=1,4}, d_{o}, G, z, \varphi, \omega\right)$. To simplify the notation, the set of 12 parameters corresponding to the four laser pulses will be denoted with $\eta=\left\{\gamma_{n}, x_{o n}, y_{o n}\right\}_{n=1,4}$. Thus the phase distribution is noted as $\Omega_{\varphi, \eta}(\bar{x})=\Omega\left(\bar{x}, \eta, d_{o}, G, z, \varphi, \omega\right)$. The retrieved phase was calculated by subtracting the phase produced by the wedge and the phase that corresponds to the region parallel to the mirror $E_{2}$. However, since the goal of this calculation is to determine whether the phase introduced by the wedge with respect to the plane is properly recovered, it was assumed, in order to avoid edge effects, that both the plane and the wedge were extended in all points of space illuminated by the beam. That is, first the phase introduced by the plane with respect to the reference mirror $E_{2}$ is calculated in every point of the detector, as if the wedge were not present (and then $\Omega_{\varphi=0, \eta}(\bar{x})$ ). Then, the same calculation for an inclined plane at an angle $\phi$, was done as occupying the entire object space (and then $\left.\Omega_{\varphi, \eta}(\bar{x})\right)$ and finally, both phases were subtracted. The phase introduced by the wedge was derived from the following equation

$$
R_{\varphi, \eta}(\bar{x})=\Omega_{\varphi, \eta}(\bar{x})-\Omega_{\varphi=0, \eta}(\bar{x}) .
$$

Since the size of the beam on the mirrors is about $4 \mathrm{~mm}$ in radius, the functions defined above were evaluated at $20 \times 10^{4}$ pixels, corresponding to the number of pixels on the detector that are covered by the beam (a pixel size of $10 \mu \mathrm{m}$ was supposed). Thus, by replacing the continuous variables $\{x, y\}$ with the discretized versions $\left\{x_{i}, y_{j}\right\}_{i, j=1: M}$ in the expressions for the retrieved phases, the phases in matrix notation were obtained as $\Omega_{\varphi=0, \eta}^{i, j}=\Omega_{\varphi=0, \eta}\left(x_{i}, y_{j}\right), \Omega_{\varphi, \eta}^{i, j}=\Omega_{\varphi, \eta}\left(x_{i}, y_{j}\right)$ and $R_{\varphi, \eta}^{i, j}=R_{\varphi, \eta}\left(x_{i}, y_{j}\right)$.

As is usually done in these techniques [43], the errors are calculated using the criterion of the root mean squared (rms), which is a statistical quantity that represents adequately the performance of the system, since it is an evaluation of all the area of measurement. The expression for the rms error is

$$
\zeta \Omega_{\varphi, \eta}=\left(\frac{\sum_{i, j}\left(\Omega_{\varphi, \eta}^{i, j}-\Omega_{\varphi, \eta_{o}}^{i, j}\right)}{N}\right)^{1 / 2}
$$

where $\Omega_{\varphi, \eta_{o}}^{i, j}$ is the phase value in the position $\left\{x_{i}, y_{j}\right\}$ for a wedge tilted by $\varphi$ in the ideal case when there are not fluctuations in the laser beam (which corresponds to the set $\eta_{o}=\{0,0,0\}$ ) and $\Omega_{\varphi, \eta}^{i, j}$ is the phase recovered in the same position but taking into account the fluctuations.

The rms errors as a function of the fluctuations of the parameters $\eta$ were finally analyzed. To simulate the shot-to-shot instability for the different pulses random numbers were generated for each parameter of the set $\eta=\left\{\gamma_{n}, x_{o n}, y_{o n}\right\}$ within tolerance intervals, using an uniform statistics distribution. This provides $\left\{x_{o n}\right\}_{n=1,4}$ varying around its zero mean value within the interval $\left(-\Delta x_{o},+\Delta x_{o}\right)$ and $\left\{\gamma_{n}\right\}_{n=1,4}$ varying around its zero mean value within the interval $(-\Delta \gamma,+\Delta \gamma)$. The absolute error $\zeta \Omega_{\varphi, \eta}$ as a function of the tolerances in the beam direction due to a pointing instability $(\Delta \gamma)$ and fluctuations in the emission axis $\left(\Delta x_{o}\right)$ were analyzed. A statistical study of the retrieved phases for different sets $\eta$ within the tolerance intervals was performed to evaluate the errors.

Figure 4 shows the results of this simulation, where the errors corresponding to the phase reconstruction of the reference plane $(\varphi=0)$ and those corresponding to the wedge $\left(\varphi=5 \times 10^{-6}\right) \mathrm{rad}$ are plotted. In both cases the influence of the telescope described in the previous section was analyzed, that is, the results are shown for beam divergences $\theta=0.005 \mathrm{rad}$ and $\theta=0.001 \mathrm{rad}$, the latter being for a telescope with 50X magnification. The maximum ranges of pointing stability values analyzed correspond to errors in the phase reconstruction of the order of $\lambda / 10$, since larger values do not meet the accuracy requirements usually set for these kinds of interferometric measurements [43].

As it was expected, the rms error increases as the fluctuations increase in all the cases, however, it can be seen that the interferometer is more sensitive to fluctuations in the incident angle than in the emission axis position. On one hand, when the fluctuations are due to translations of the emission axis $x_{o}$, no significant differences are observed for different angles $\varphi$. The use of the expander and collimator system decreases the error by a factor 

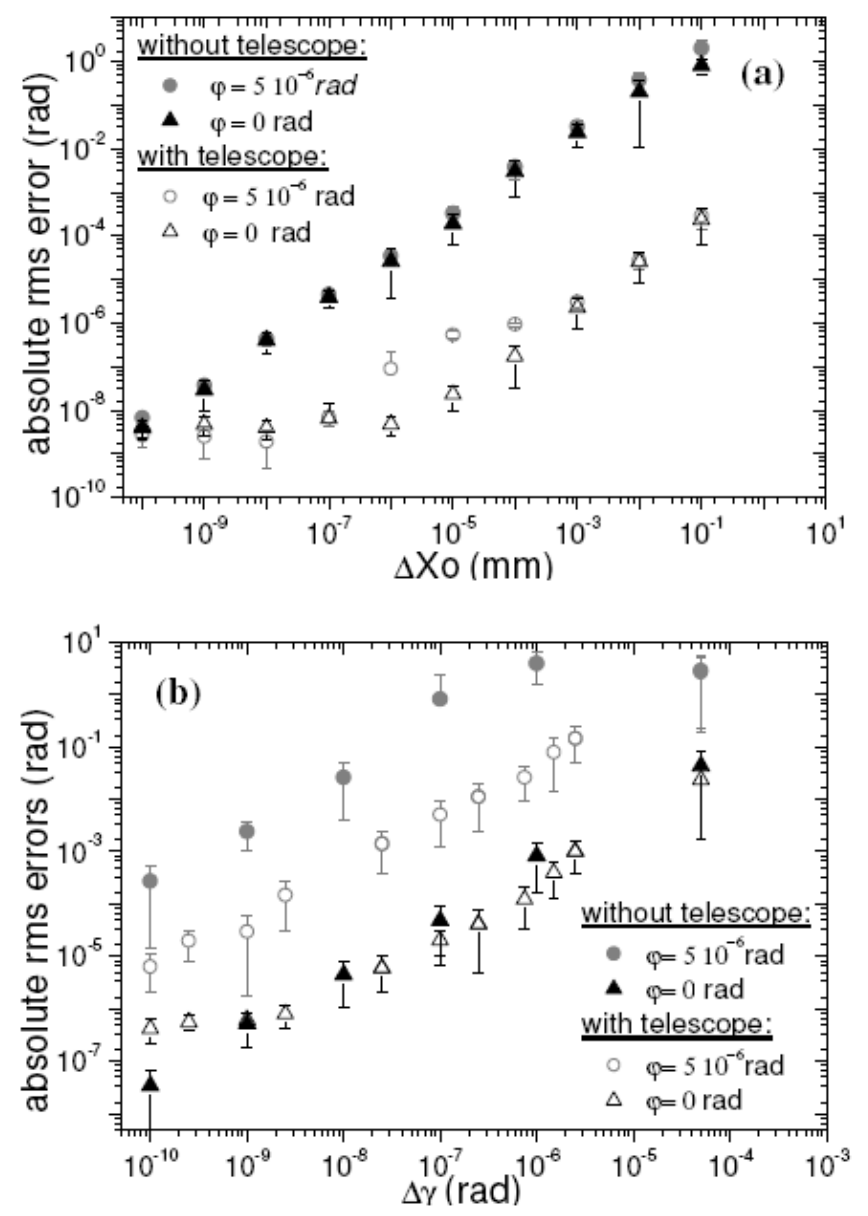

Fig. 4. Graph comparing the absolute error in the phase retrieving for a tilt angle equal to $5 \times 10^{-6} \mathrm{rad}$ (gray) and without tilt (violet) as a function of the tolerances, for all the simulations. (a) the fluctuating parameter is the emission axis $x_{o}$. (b) the fluctuating parameter is the incidence angle $\gamma$

of up to four orders of magnitude. On the other hand, figure 4 shows that for fluctuations in the incidence angle, the error in the determination of the phase corresponding to $\varphi=0$ is four orders of magnitude smaller than for the tilted wedge. In the latter case, the telescopic system decreases the error by two orders of magnitude. It is expected that the higher magnification the smaller the errors because the wavefront becomes increasingly flatter through the optical paths. Remembering that the plane $\varphi=0$ is parallel to the mirror in the reference arm of the interferometer, it is clear that such small fluctuations in the angle of incidence increases when the laser impinge on areas that are deviated from this plane.

Since in all the cases the error in the determination of the phase corresponding to $\varphi=0\left(\zeta \Omega_{\varphi=0, \eta}\right)$
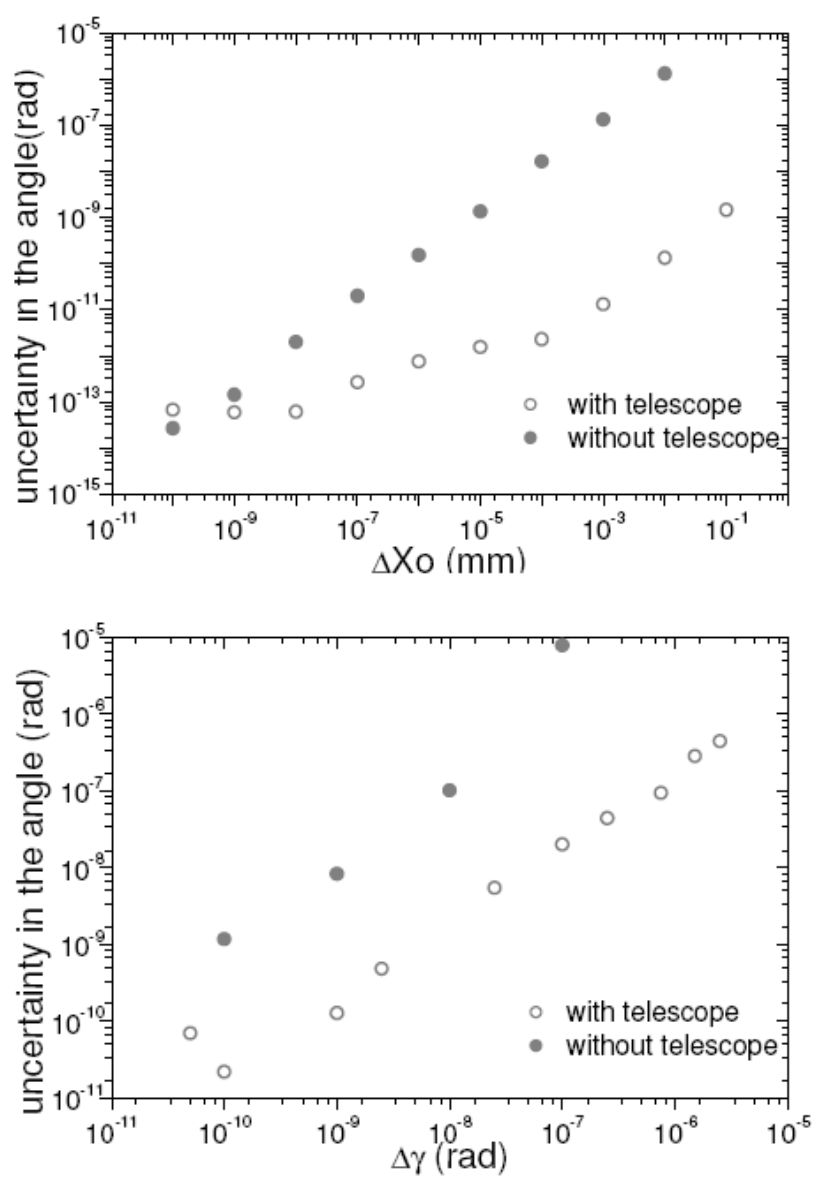

Fig. 5. Uncertainty in the recovery of the inclination of the wedge in terms of the tolerances on the fluctuations for an average of the reconstructed phase. The tilt angle is $\varphi=5 \times 10^{-6} \mathrm{rad}$

is several orders of magnitude smaller than for the tilted wedge $\left(\zeta \Omega_{\varphi, \eta}\right)$ (see figure 4$)$, the error in the retrieved phase $\left(\zeta R_{\varphi, \eta}\right)$ is approximately equal to $\left(\zeta \Omega_{\varphi, \eta}\right)$ and the precision is approximately equal to the precision in measuring $\left(\Omega_{\varphi}\right)$.

The beam splitters of the interferometer are implemented with diffraction gratings, thus it should be expected that the alignment will be influenced by the bandwidth of the source used for the experiment. This effect is expected to be more important if a HHG source is used, due to its larger bandwidth as compared with EUV lasers. However, even for these sources $(\Delta \lambda / \lambda=0.1)$, the influence is negligible, yielding a maximum optical path difference less than $3.4 \times 10^{-5}$ across the spectrum.

Finally from the phase distributions the tilt angle of the wedge can be retrieved averaging over all simulated data obtained for each tolerance interval, in the situations discussed throughout this paper. Figure 5 shows the data for errors in the recovery of 
the inclination angle as a function of the tolerance for a wedge angle of $\varphi=5 \times 10^{-6} \mathrm{rad}$, for the cases where it is used a telescope system or a direct illumination on the grating. The cases in which fluctuations are due to pointing instabilities $(\Delta \gamma)$ and when they are due to variations in the laser emission axis $\left(\Delta x_{o}\right)$ were analyzed.

The error increases when fluctuations increase. For the range of the acceptable instabilities discussed previously (those that introduced errors below $\lambda / 10)$ the values obtained for the inclination of the wedge were correct. However, comparing these results with those obtained in figure 5 , in the case of not using an expander and collimator system, it is noted that the limit values obtained in the phase error (values close to $\lambda / 10$ ) corresponds to errors in the tilt angle greater than or equal to the value of tilt to be measured, therefore the measurement in such cases would not be appropriate. As expected, the use of the expander and collimator system leads to the decrease of the error by several orders of magnitude, allowing the system to be more tolerable to the laser fluctuations.

\section{Conclusion.}

In this work it is proposed an interferometer suitable for working in the extreme ultraviolet and useful to implement the PSI technique. The illumination source considered in this design is a table top capillary discharge EUV laser. The instrument was designed using reflective and diffractive optics compatible with the EUV illumination. The proposed two beam interferometer is composed by two gratings and two mirrors that provide completely symmetrical paths. The phase shifts required for applying the PSI technique, are obtained by moving across one of the gratings. In order to perform a study of the influence of the pointing fluctuations that the laser suffers from shot to shot an algorithm capable of simulating the propagation of the laser beam in the device was derived, considering that each interferogram must be acquired with a differ- ent pulse. The phase retrieval was numerically performed taking into account the construction parameters of the interferometer and the features of the light source. To this end a test object was used composed by a wedge with an inclination of $\varphi=5 \times 10^{-6}$ $\mathrm{rad}$. The correct recovering of the phase introduced by this object was analyzed as a function the errors caused by fluctuations in the incidence angle and fluctuations in the positioning of the emission axis of the laser. Two different cases were studied; one corresponds to using a non collimated laser beam and the other employing a collimator system with $50 \times$ magnification. As it was expected, for all cases it is observed an increasing error as the fluctuations increased. In addition, it is found that the device is more sensitive to fluctuations in the angle of incidence than to displacements of the emission axis. On the other hand it is confirmed that the use of the telescope produces a decrease in the errors by a factor greater than two orders of magnitude. The comparison between the angle of inclination of the wedge with its error, revealed that the limiting values allowed for the fluctuations in the laser emission axis, is of the order of $\Delta x_{o}=10^{-2} \mathrm{~mm}$, while in the case of the fluctuations in the emission angle results to be $\Delta \gamma<10^{-7}$ rad since for higher tolerances the uncertainty in determining the angle does not make reliable measurement. As expected, the expander and collimator system decreases the error by several orders of magnitude, which means that an appropriate magnification can be found in order to compensate the actual laser fluctuations. In order to decrease the error in the phase retrieval calculation, the confocal parameter can be outstretched using a telescope or the interferometer's optical path can be shortened designing a smaller foot print instrument.

\section{Appendix A: Amplitude of the electric field}

In this appendix explicit expressions for $A_{1}\left(\bar{x}, l_{2}, l_{1}, d, \omega\right)$ and $A_{2}\left(\bar{x}, s_{2}, s_{1}, d, \omega\right)$ are given.

In the case of the arm 1 , it is obtained 


$$
\begin{array}{r}
A_{1}\left(\bar{x}_{4}^{\prime}, l_{2}, l_{1}, d, \omega\right)=-i z_{r} A_{o} S_{R}(\omega) B \sqrt{\frac{1}{q\left(d+l_{1}+l_{2}+z_{4}^{\prime}\right) q\left(d+\alpha_{1}^{2}\left(l_{1}+l_{2}+z_{4}^{\prime} \alpha_{2}^{2}\right)\right)} \times} \\
\exp \left(-\frac{i k_{o}}{2}\left(2\left(d+l_{1}+l_{2}+z_{4}^{\prime}\right)+\frac{x_{4}^{\prime 2}}{z_{4}^{\prime}}+\frac{x_{o}^{\prime 2}}{q(d)}+\frac{\left(y_{4}^{\prime}-y_{o}^{\prime}\right)^{2}}{q\left(d+l_{1}+l_{2}+z_{4}^{\prime}\right)}-\frac{l_{1}\left(x_{o}^{\prime} \alpha_{1}+q(d) \beta_{1}\left(\omega-\omega_{o}\right)\right)^{2}}{q(d) q\left(d+\alpha_{1}^{2} l_{1}\right)}\right)\right) \times \\
\exp \left(-\frac{i k_{o}}{2} \frac{\alpha_{1}\left(-x_{o}^{\prime} z_{4}^{\prime} \alpha_{2}+\alpha_{1}\left(l_{1}+l_{2}\right)\left(x_{4}^{\prime}+z_{4}^{\prime} \beta_{2}\left(\omega-\omega_{o}\right)\right)+\left(x_{4}^{\prime}-z_{4}^{\prime}\left(\alpha_{2} \beta_{1}-\beta_{2}\right)\left(\omega-\omega_{o}\right)\right) q(d)\right)^{2}}{z_{4}^{\prime} q\left(d+\alpha_{1}^{2}\left(l_{1}+l_{2}\right)\right) q\left(d+\alpha_{1}^{2}\left(l_{1}+l_{2}+\alpha_{2}^{2} z_{4}^{\prime}\right)\right)}\right) \times \\
\exp \left(-\frac{i k_{o}}{2} \frac{l_{2}\left(x_{o}^{\prime} \alpha_{1}+q(d) \beta_{1}\left(\omega-\omega_{o}\right)\right)^{2}}{q\left(d+\alpha_{1}^{2}\left(l_{1}+l_{2}\right)\right) q\left(d+\alpha_{1}^{2} l_{1}\right)}\right) \exp \left(i \phi_{+1}\right)
\end{array}
$$

where $\alpha_{i}$ and $\beta_{i}$ are the angular and frequency dispersion coefficients of the gratings $i=1$ and $i=2$ for the arm 1, and are obtained as indicated in ref [46]. The coefficient $B$ includes the transmission efficiency of both gratings in the order 1 and the mirror reflectivity. $q\left(z+i z_{r}\right)$ is the complex radius of curvature of a Gaussian beam, where $z_{r}$ is the Raleigh parameter. $\phi_{+1}$ is the phase shift added due to the relative translation of the gratings and the light beam previously described. The electric field is written in the natural coordinate system $S_{4}^{\prime}$ given by the coordinates $x_{4}^{\prime} y_{4}^{\prime} z_{4}^{\prime}$, that corresponds to the section that extends from the second grating to the detector. Then, in order to get an expression in the laboratory system $S$, both systems are related by using a linear transformation given by $\bar{x}_{4}^{\prime}=\bar{x}_{c}^{\prime}(0)+R^{-1}\left(\theta_{1}^{G_{2}}\right)\left(\bar{x}-\bar{x}_{c}\left(G+d_{o}\right)\right)$ where $\bar{x}_{c}^{\prime}(0)$ and $\bar{x}_{c}\left(G+d_{o}\right)$ are the coordinates of a reference point on the second grating written in both systems and $R^{-1}\left(\theta_{1}^{G_{2}}\right)$ is the inverse of a rotation matrix around the y coordinate in an angle equal to the first order diffraction angle in the second grating $\theta_{1}^{G_{2}}$, which is a function of the diffraction angle on the first grating $\theta_{1}^{G_{1}}$, the tilt of the mirror $\varphi$, and the period of the grating $p$.

The corresponding expressions of the arm -1 , are obtained in complete analogy to that of the arm 1. So they can be obtained by substitution of the propagation distances and the grating coefficients of the arm 1 by the corresponding to the arm -1 in the expression A1. That is to say, $l_{1}$ is replaced by $s_{1}, l_{2}$ by $s_{2}, \alpha_{1}$ by $\alpha_{-1}, \beta_{1}$ by $\beta_{-1}$ and the same for the second grating coefficients, $\phi_{+1}$ by $\phi_{-1}$ and $\varphi=0$.

\section{Acknowledgments}

This work was supported by grants from Buenos Aires University (20020100100689 and 20020110300027) and a grant from ANPCyT (grant PICT Bicentenario 2010-02169). MGC and
CCI are members of CONICET. MCM acknowledges the support from the NSF ERC for Extreme Ultraviolet Science and Technology, award EEC 0310717.

\section{References}

[1] D. H. Martz, D. Alessi, B. M. Luther, Y. Wang, D. Kemp, M. Berrill, and J. J. Rocca, "High-energy $13.9 \mathrm{~nm}$ table-top soft-x-ray laser at $2.5 \mathrm{~Hz}$ repetition rate excited by a slab-pumped Ti:sapphire laser," Opt. Lett. 35, 1632-1634 (2010).

[2] D. Alessi, D. H. Martz, Y. Wang, M. Berrill, B. M. Luther, and J. J. Rocca, "Gain-saturated $10.9 \mathrm{~nm}$ tabletop laser operating at $1 \mathrm{~Hz}$ repetition rate," Opt. Lett. 35, 414-416 (2010).

[3] D. A. Alman, H. Qiu, T. Spila, K. C. Thompson, E. L. Antonsen, B. E. Jurczyk and D. N. Ruzic, "Characterization of collector optic material samples exposed to a discharge-produced plasma extreme ultraviolet light source," Journal of Micro/Nanolithography, MEMS, and MOEMS 6 (1), 013006 (2007).

[4] M. Tanaka, Y. Furukawa, H. Murakami, S. Saito, N. Sarukura, M. Nishikino et al, "Evaluation of fast EUV scintillator using $13.9 \mathrm{~nm}$ x-ray laser," The fifth International Conference on Inertial Fusion Sciences and Applications (IFSA2007) 112, 042058 (2008).

[5] L. Juha, M. Bittner, D. Chvostova, J. Krasa, Z. Otcenasek, A. R. Prg et al, "Ablation of organic polymers by 46.9-Nm-Laser radiation," Applied Physics Letters 86 (3), 034109 (2005).

[6] I. A. Artioukov, B. R. Benware, J. J. Rocca et al, "Determination of XUV optical constants by reflectometry using a high-repetition rate $46.9 \mathrm{~nm}$ laser," IEEE Journal Of Selected Topics In Quantum Electronics 5 (6), 1495-1501 (1999).

[7] D. T. Attwood, G. E. Sommargren, R. Beguiristain, K. Nguyen and J. Bokor, "Undulator radiation for at-wavelength interferometry of optics for extreme-ultraviolet lithography," Applied Optics 32 
(34), 7022 (1993).

[8] P. P. Naulleau and K. A. Goldberg, "Dual-domain point diffraction interferometer", Applied Optics 38 (16), 3523-3533 (1999).

[9] H. Medecki, E. Tejnil, K. A. Goldberg and J. Bokor, "Phase-shifting point diffraction interferometer," Optics Letters 21, 1526-1528 (1996).

[10] G. E. Sommargren, "Phase Shifting Difraction Interferometer for Measuring Extreme Ultraviolet Optics," in Optical Society of America meeting on extreme ultraviolet lithography (1996).

[11] P. P. Naulleau, K. A. Goldberg, S. H. Lee, C. Chang, D. Attwood and J. Bokor, "Extremeultraviolet phase-shifting point-diffraction interferometer: a wave-front metrology tool with subangstrom reference-wave accuracy", Applied Optics 38 (35), 7252-7263 (1999).

[12] E. T. Kennedy, J. T. Costello, J. P. Mosnier and P. van Kampen, "VUV/EUV ionising radiation and atoms and ions: dual laser plasma investigations," Radiation Physics and Chemistry 70 (1-3), 291321(2004).

[13] Y. Hatano, "Spectroscopy and dynamics of molecular superexcited states. Aspects of primary processes of radiation chemistry," Radiation Physics and Chemistry 67 (3-4), 187-198 (2003).

[14] D. T. Attwood, E. Anderson, G. Denbeaux, K. Goldberg, P. Naulleau and G. Schneider, "Soft XRay Microscopy and EUV Lithography: An Update on Imaging at 20-40 nm Spatial Resolution," X-RAY LASERS 2002: 8th International Conference on X-Ray Lasers AIP Conf. Proc. 641, 461-468 (2002).

[15] R. N. Watts, T. B. Lucatorto, S. T. Liang, F. Polack, and M. R. Scheinfein, "EUV and soft x-ray transmission microscope," Rev. Sci. Instrum. 67, 3359 (1996).

[16] D. Shapiro, P. Thibault, T. Beetz, V. Elser et al "Biological imaging by soft x-ray diffraction microscopy" PNAS 102 (43) 15343-15346 (2005).

[17] H. Jiang, C. Song, C. Chen, R. Xu, K. S. Raines et al "Quantitative 3D imaging of whole, unstained cells by using X-ray diffraction microscopy," PNAS 107 (25) 11234-11239 (2010).

[18] J. Filevich, K. Kanizay, M. C. Marconi, J. L. Chilla and J. J. Rocca, "Dense plasma diagnostics with an amplitude-division soft-x-ray laser interferometer based on diffraction gratings," Optics Letters 25 (5), 356-358 (2000).

[19] J. Filevich, J. J. Rocca, M. C. Marconi, S. J. Moon, J. Nilsen, J. H. Scofield, J. Dunn, R. F. Smith, R. Keenan, J. R. Hunter and V. N. Shlyaptsev, "Observation of a multiply ionized plasma with index of refraction greater than one," Phys. Rev. Lett. 94 (3), 035005 (2005).

[20] M. A. Purvis, J. Grava, J. Filevich, M. C. Mar- coni, J. Dunn, S. J. Moon, V. N. Shlyaptsev, E. Jankowska and J.J. Rocca, "Soft X-ray laser interferometry of colliding laser-created plasmas in semicylindrical cavities," IEEE Transactions on Plasma Science 36, 1134-1135 (2008).

[21] J. J. Rocca, C. H. Moreno, M. C. Marconi and K. Kanizay, "Soft-x-ray lser interferometry of a plasma with a tabletop laser and a Lloyd's mirror," Optics Letters 24 (6), 420-422 (1999).

[22] P. L. Shkolnikov and A. E. Kaplan, "Feasibility of x-ray resonant nonlinear effects in plasmas," Opt. Lett. 16, 1153-1155 (1991).

[23] T. Popmintchev, M. Chen, P. Arpin, M. M. Murnane and H. C. Kapteyn "The attosecond nonlinear optics of bright coherent X-ray generation," Nature Photonics 4, 822-832 (2010).

[24] C. Wagner and N. Harned "EUV lithography: Lithography gets extreme," Nature Photonics 4, 24 - 26 (2010).

[25] J. E. Bjorkholm, A. A. MacDowell, O. R. Wood II, Z. Tan, B. LaFontaine, and D. M. Tennant. "Phasemeasuring interferometry using extreme ultraviolet radiation," J. Vac. Sci. Technol. B 13, 2919-2922 (1995).

[26] C. D. Macchietto, B. R. Benware, and J. J. Rocca, "Generation of millijoule-level soft-x-ray laser pulses at a $4-\mathrm{Hz}$ repetition rate in a highly saturated tabletop capillary discharge amplifier," Optics Letters, 24(16),1115-1117(1999)

[27] B. R. Benware, C. D. Macchietto, C. H. Moreno, and J. J. Rocca, "Demonstration of a High Average Power Tabletop Soft X-Ray Laser," Phys. Rev. Lett.81(26), 58045807 (1998)

[28] Y. Liu, M. Seminario, F. G. Tomasel, C. Chang, J. J. Rocca and D. T. Attwood, "Achievement of essentially full spatial coherence in a high-averagepower soft-X-ray lser," Phys. Rev. A, Gen. Phys. 63, 033802 (2001).

[29] L. Urbanski, M. C. Marconi, L. M. Meng, M. Berrill, O. Guilbaud, A. Klisnick, J. J. Rocca, "Spectral linewidth of a Ne-like Ar capillary discharge soft $\mathrm{x}$-ray laser and its dependence on amplification beyond gain-saturation," Physical Review A 85, 033837 (2012).

[30] M. C. Marconi, J. L. A. Chilla, C. H. Moreno, B. R. Benware and J. J. Rocca, "Measurement of the Spatial Coherence Buildup in a Discharge Pumped Table-Top Soft X-Ray Laser," Phys. Rev. Lett. 79, 2799-2802 (1997).

[31] J. L. A. Chilla, J. J. Rocca, O. E. Martinez and M. C. Marconi, "Soft-x-ray interferometer for singleshot laser linewidth measurements," Optics Letters 21 (13), 955-957 (1996).

[32] J. Filevich, J. J. Rocca, E. Jankowska, E. C. Hammarsten, K. Kanizay, M. C. Marconi et al, "Twodimensional effects in laser-created plasmas mea- 
sured with soft-x-ray lser interferometry," Physical Review E 67 (5), 2-7(2003).

[33] M. G. Capeluto, G. Vaschenko, M. Grisham, M. C. Marconi et al, "Nanopatterning with interferometric lithography using a compact $\lambda=46.9$-nm laser," IEEE Transactions on Nanotechnology 5 (1), 3-7 (2004).

[34] E. B. Malm, N. Monserud, C. G. Brown, P. W. Wachulak, H. Xu, G. Balakrishnan, W. Chao, E. Anderson and M. C. Marconi. "Tabletop single-shot extreme ultraviolet Fourier transform holography of an extended object," Optics Express 21, 9959, (2013).

[35] L. Urbanski, A. Isoyan, A. Stein, J. J. Rocca, C. S. Menoni, M. C. Marconi, "Defect Tolerant Extreme Ultraviolet Nanoscale Printing," Optics Letters 37 3633, (2012).

[36] C. S. Menoni, F. Brizuela, I. Howlett, S. Carbajo, D. Peterson, M. C. Marconi, J. J. Rocca, A. Sakdinawat, Y. Liu, D. T. Attwood, "Imaging at the nanoscale with practical table-top EUV full field microscopes," IEEE Selected topics on Quantum Electronics 18 434, (2011).

[37] H. Bravo, B. T. Szapiro, P. W. Wachulak, M. C. Marconi, W. Chao, E. H. Anderson, C. S. Menoni, J. J. Rocca, "Demonstration of Nanomachining with Focused Extreme Ultraviolet Laser Beams," IEEE Selected topics on Quantum Electronics, 18
443, (2011).

[38] S. Carbajo, I. Howlett, F. Brizuela, K. Buchanan, M. Marconi, W. Chao, E. Anderson, I. Artioukov, A. Vinogradov, J. Rocca, and C. Menoni, "Sequential single-sh ot imaging of nanoscale dynamic interactions with a table-top soft x-ray laser," Opt. Lett. 37, 2994-2996 (2012).

[39] D. Malacara, Optical Shop Testing, (John Wiley and Sons, New York, 3th Edition, 2007).

[40] J. C. Wyant, "Interferometric optical metrology: basic principles and new systems," Laser Focus 18, 65-71 (1982).

[41] P. J. de Groot "Vibration in phase-shifting interferometry," J. Opt. Soc. Am. A 12 (2) 354-365 (1995)

[42] J. E. Greivenkamp and J. H. Burning "Phase shifting interferometry" in Optical Shop Testing, D. Malacara editor (New York: Wiley,1992) 501-598.

[43] K. Creath, "Chapter V: Phase-Measurment Interferometry Techniques," in Progress in Optics XXVI, Wolf editor (Elsevier Science Publishers B.V.,1998), 349-393.

[44] A. D. Siegman, Lasers, (University Science Books, Mill Valley, CA, 1986), 301.

[45] W. T. Silfvast, Laser Fundamentals, (Cambridge University Press, Cambridge, 1996).

[46] O. E. Martinez, "Grating and prism compressors in the case of finite beam size," Journal Of The Optical Society Of America B Optical Physics 3 (7), 929934 (1986). 